\title{
Optimization of Textured-surface Light Emitting Diode
}

\author{
E. Herbert Li, Senior Member, IEEE, Chun-Chung Chan, and Paul C. K. Kwok, Member, IEEE \\ Department of Electrical \& Electronic Engineering, The University of Hong Kong. \\ Email: ehli@eee.hku.hk
}

\begin{abstract}
We present an analysis of the efficiency and radiation pattern of the textured-surface LED by tracing the light rays that emits from the active layer. Through simulation, we discover that the efficiency depends on the thickness of the textured layer and its separation distance from the active layer. By carefully choosing these two parameters, maximum efficiency an be achieved. The radiation pattern of a texture-surface LED is found to be different from the flat-surfaced LED in that the textured surface has a wider angular spread.
\end{abstract}

This work is supported by the Hong Kong RGC earmarked grant and The University of Hong Kong CRCG grant.

\section{Introduction}

Many applications such as high intensity full color displays and optical communication systems require high intensity light emitting diodes (LED's) for better performance. The external quantum efficiency of today's compound semiconductor LED's is still very small, typically less than $10 \%$ [1], although the internal efficiency can be very high $(\sim 100 \%)$ [2]. This is because the refractive index of semiconductor materials is usually quite high $(\sim 3.5)$, more than three times as air, therefore the critical angle $\theta_{c}$ given by Snell's Law (from semiconductor to air) is very small, typically $\sim 17^{\circ}$ maximum. Only the light generated in the active region that strikes at the LED surface within the critical angle can escape the device. The rest of the light suffers from total internal reflection and eventually reabsorbed within the semiconductor. The small critical angle becomes the limiting factoring affecting the efficiency of an LED. If we can improve the external quantum efficiency, the overall efficiency of LED's can be increased substantially.

Besides choosing a material of lower refractive index, the geometrical design of the LED's surface also plays an important role in enhancing the external quantum efficiency. The commercial design is a flat surface or an epoxy doomed structure and has a low efficiency.

Recently, LED's having external quantum efficiency as high as $30 \%$ has been reported [3]; this was achieved by fabricating a coarse texturing on the semiconductor surface, where the escape probability of photons can be increased substantially. However, the mechanism of achieving such a high efficiency due to the textured surface is not yet fully understood. We will show that the enhanced efficiency is due to the random nature of the textured surface which provides multiple opportunities for light to escape. It has a greater escape cone than the flat surface; thus the probability for light to escape is higher.

\section{Modeling of the Textured Surface}

The textured window layer in the LED can be visualized as having two homogeneous parts. The top part is a rough layer of thickness $x$, enclosing the highest and lowest point of the surface (Figure 1). The bottom part is the bulk of the transparent material of thickness $L$ and it extends form the active layer to the lowest point of the textured surface.
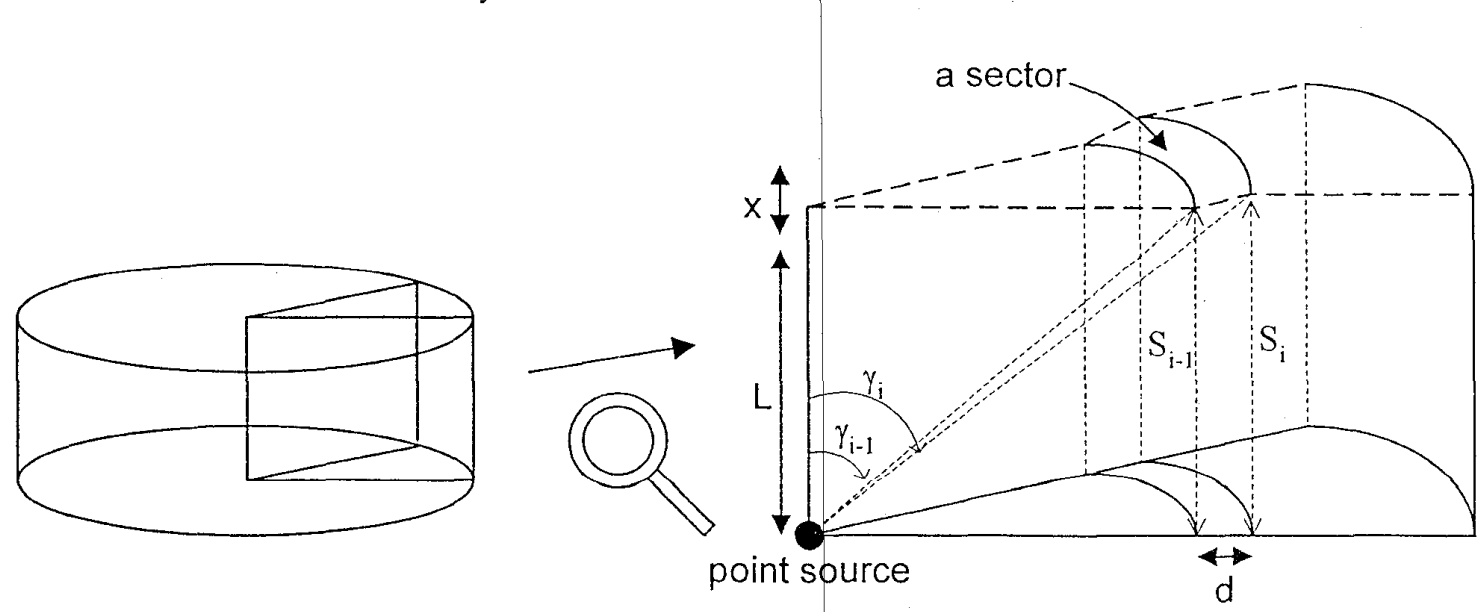

Figure 1. Model of the textured-surface LED using a cylinder 
We model the structure as an upright cylinder. The top face of the cylinder is the textured surface and the bottom face is the active layer. The cylinder is divided into concentric tracks and each track is divided into a large number of sectors. Each sector is modeled as having a top surface that is slanted and planar, with a random inclination. For the purpose of analysis it is sufficient to just consider a single point source at the centre of bottom surface of the cylinder. The total intensity of light emitted can be expressed as the sum of intensities from an ensemble of point sources evenly distributed on the active surface.

The light rays emitted from the point source are traced. At the point the ray hits the surface in a particular sector, the angle of incidence is calculated and compared with the critical angle to determine if the ray can escape through the surface. The range of angles of the light rays that are able to travel through the surface of a sector is called the escape angle. The total intensity emitted from the entire textured surface is related to the sum of the escape angles for all sectors. In our analysis, we also take into account of the Fresnel reflection loss.

\section{Simulation, Result and Analysis}

The sectored model described in the previous section can be used for simulation to estimate the amount of light that emits from the textured surface. During simulation, a random number generator is used to obtain $\mathrm{S}_{\mathrm{i}}$, the heights of the limiting points of the sectors (Figure 1). The following values for various parameters are found to be adequate for the purpose of the simulation. The width of a track $\mathrm{d}$ is $5 \mu \mathrm{m}$. The total number of tracks, $\mathrm{N}$ is equal to 1001 , making a radius of $500 \mu \mathrm{m}$ for the LED. Light rays emitted from the point source at intervals of $0.01^{\circ}$ are traced to determine the angle of incidence at the surface. The escape angle for each sector can then be determined. The external quantum efficiency and radiation pattern of the LED are determined and studied as a function of (a) the thickness of the textured surface $\mathrm{x}$ between $0.1 \mu \mathrm{m}$ to $10 \mu \mathrm{m}$; (b) the height between the active layer and the bottom of the textured surface $\mathrm{L}$ between $0.01 \mu \mathrm{m}$ to $100 \mu \mathrm{m}$. The results are discussed below.

\section{A. Thickness of textured surface, $x$}

Figure 2 shows the variations of external quantum efficiency as a function of $x$ and L. As $x$ increases from $0.1 \mu \mathrm{m}$, the external quantum efficiency increases gradually for all values of $\mathrm{L}$ and attains a maximum when $\mathrm{x}$ is about $1 \mu \mathrm{m}$. It begins to decrease as $\mathrm{x}$ is further increased, and beyond $10 \mu \mathrm{m}$ the efficiency becomes relatively constant. The explanation is as follows. Considering a single sector in our model (Figure 4a). The inclination $\alpha$ is allowed to rotate within the boundary of $x$. The incident angle $\phi$ must be smaller than the critical angle $\theta_{c}$ in order to let the light pass through. Let $\mathrm{L}=1 \mu \mathrm{m}$. Initially, in Figure 4 (a) case (i) when $x=x_{1}$, the surface is denoted as at position $1 . \phi$ is greater than $\theta_{c}$, leading to lower efficiency. As $x$ increases to $x_{2}$ (case (ii)), $\alpha$ increases and the surface is more inclined from position 1 to position 2. $\phi$ is now becoming smaller and smaller so that when $\phi<\theta_{c}$, light begins to enter the escape cone. Hence the efficiency increases gradually with $x$. However as $x$ is further increased to $x_{3}$ (case (iii)), the surface inclination changes from position 2 to position 3 . $\phi$ is once again larger than $\theta_{c}$, and light begins to stay outside the escape cone. As a result the efficiency starts to decrease when $x$ is greater than $1 \mu \mathrm{m}$ for the structure considered here.

\section{B. Height between the active layer and the bottom of the textured surface \\ The external quantum efficiency shows a} similar trend as $L$ varies (Figure 2). Let $x=1 \mu \mathrm{m}$. The efficiency increases as $\mathrm{L}$ increases from $0.01 \mu \mathrm{m}$ onwards and attains its maximum at $1 \mu \mathrm{m}$, it then decreases as $\mathrm{L}$ is increased further to $100 \mu \mathrm{m}$. The phenomenon can be explained in a similar manner. We fix $x$ so that the inclination $\alpha$ is allowed to rotate within the limit of $\alpha=0$ and $\tan ^{-1}(\mathrm{x} / \mathrm{d})$ only. When $\mathrm{L}=\mathrm{L}_{1}$ as shown in Figure 4 (b) case (i), $\phi$ is greater than $\theta_{c}$ leading to lower efficiency. Then as $L$ increases to $L_{2}$ (case (ii)), $\phi$ falls within the escape cone. As $L$ increases further to $\mathrm{L}_{3}$ (case (iii)), $\phi$ begins to fall outside the escape cone. As a result the efficiency increases and then decreases when $L$ is changed from $L_{1}$ to $L_{2}$ and then from $L_{2}$ to $L_{3}$. Refer to Figure 2 again, for the cases of $x=1 \mu \mathrm{m}$ and $0.4 \mu \mathrm{m}$. It shows that as $\mathrm{L}$ increases from $0.01 \mu \mathrm{m}$ onwards, the escape probability increases gradually to reach a maximum value, then decreases again.

\section{Ratio of $x$ to $L$}

Figure 3 illustrates an interesting property regarding $z$, the ratio of $x$ to $L$. As $z$ increases, the attainable maximum efficiency also increases. The efficiency is highest between $\mathrm{L}=0.5 \mu \mathrm{m}$ and $1 \mu \mathrm{m}$. Moreover, the efficiency becomes "saturated" around $\mathrm{L}=1 \mu \mathrm{m}$. Thus illustrates that during the design of textured surface LED, the values of $x$ and $L$ can be optimized to achieve the maximum efficiency.

\section{Radiation Pattern}

The high radiance of surface emitting LED is achieved by confining the emission to a very small area [5]. The radiation pattern of a typical flat-surfaced LED has a narrow main lobe in the normal direction (Figure 5 ). However, the radiation pattern of the texture-surfaced LED is more wide spread as shown in Figure 6. This is consistent with the fact that the power emitted, or the external quantum efficiency, is much higher in the textured-surface LED than in the flat-surfaced LED. However, the power emitted in the normal direction is lower than in other angular positions, resulting in a "butterfly" shape.

\section{Conclusion}

It is shown that in the design of high intensity LED's, substantial increase in efficiency is achieved by 
using a textured surface. The characteristics of the texture-surfaced LED's have been studied in terms of its external quantum efficiency and radiation pattern. A detailed description of the mechanism involved has been presented. We have found that the major contribution to the enhanced efficiency is due to the randomness of the inclined façade surfaces. This efficiency is strongly dependent on the thickness of the textured surface layer and its distance to the active layer of the diode. There is an optimal range for these parameters in order to attain the maximum efficiency.

Moreover, it is found that the radiation pattern of the texture-surfaced LED has a wider spread of intensity in the angular direction than the flat surface. However, the power emitted in the normal direction is lower, resulting in a 'butterfly' shape. These results can be used as a guideline for the design of textured-surface LED's.

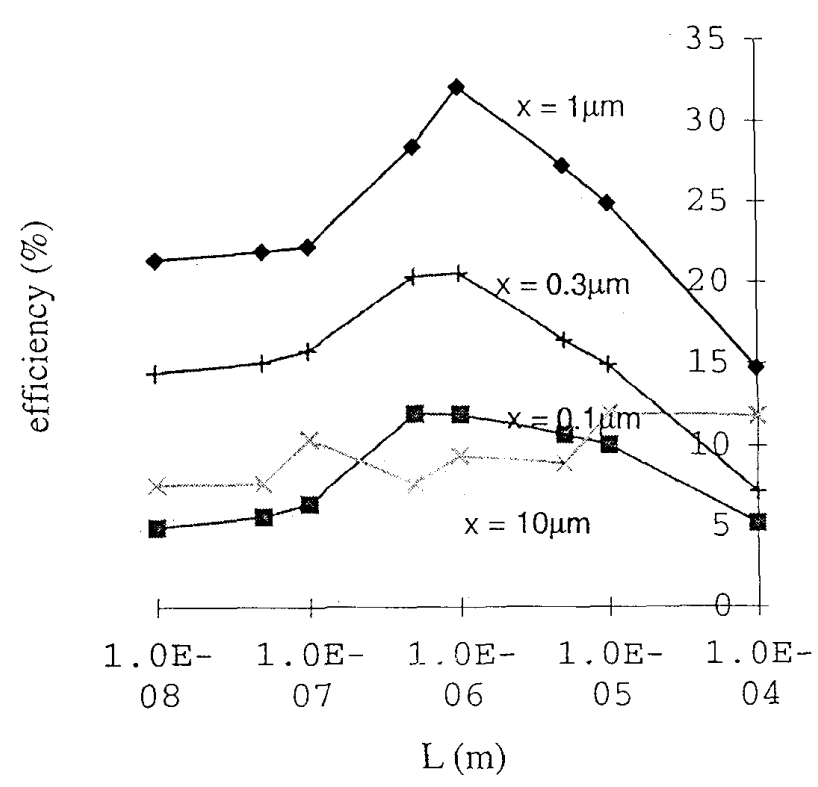

Figure 2. Efficiency as a function of $x$ and $L$

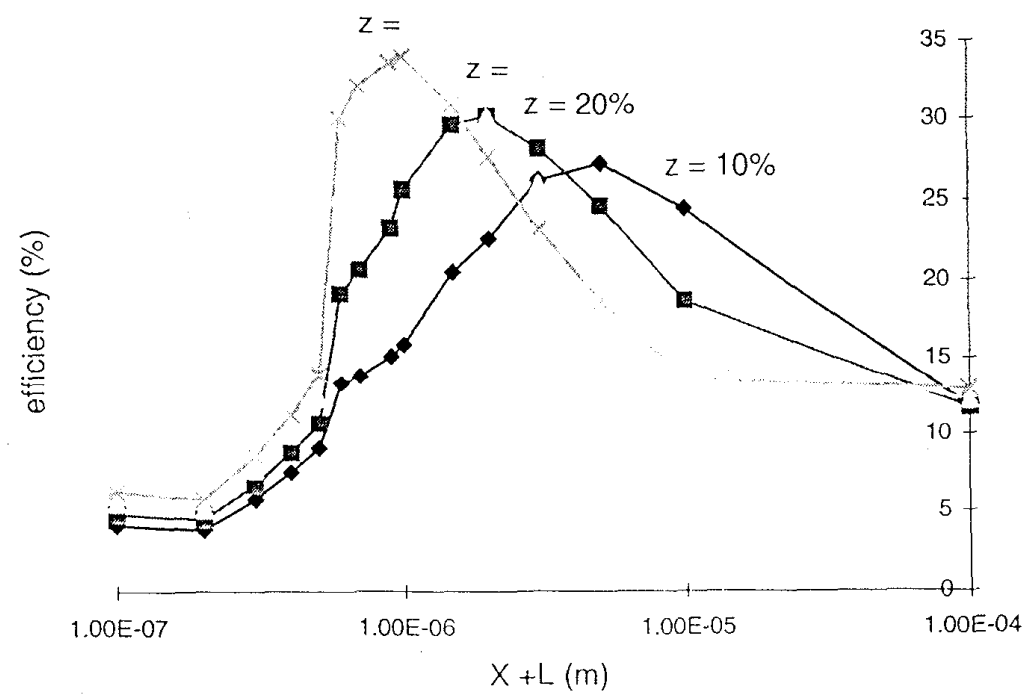

Figure 3. Efficiency as a function of $\mathrm{x}+\mathrm{L}$ and $z$.

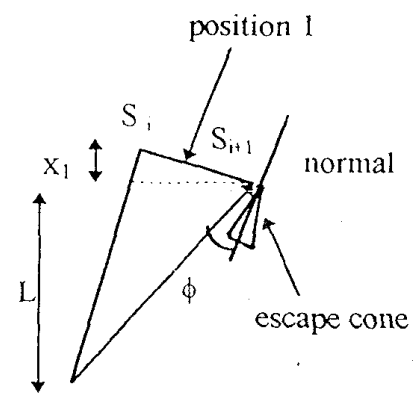

light source

Case (i)

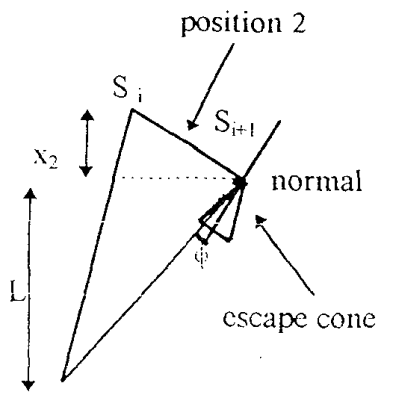

light source

Case (ii)

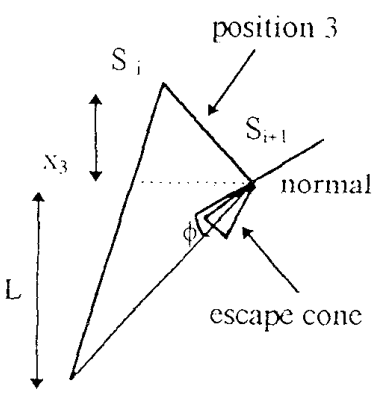

light source

Case (iii)

Figure $4 a$. The effect of varying $x$. 


\section{References}

[1] G.B. Stringfellow and M.G. Craford, Editors, $\mathrm{High}$ Brightness Light Emitting Diodes, Semiconductors and Semimetals, vol. 48, San Diego: Academic Press, 1997, Chapter 2.

[2] I. Schnitzer, E. Yablonovitch, C. Caneau, and T.J. Gmitter, "Ultrahigh spontaneous emission quantum efficiency, $99.7 \%$ internally and $72 \%$ externally, from AlGaAs/GaAs/AlGaAs double heterostructures", Appl. Phys. Lett., vol. 62, p.131133, 1993.

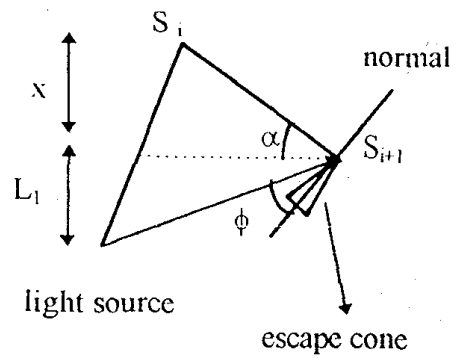

\section{Case (i)}

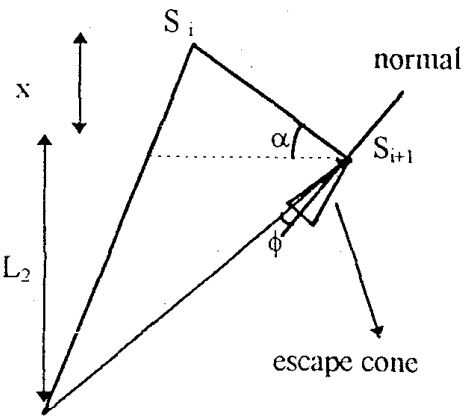

light source

Case (ii)

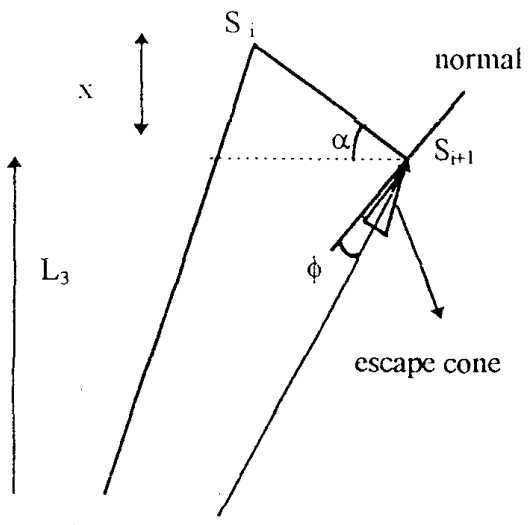

light source

Case (iii)

Figure $4 \mathrm{~b}$. The effect of varying $\mathrm{L}$
[3] I. Schnitzer, E. Yablonovitch, C. Caneau, T.J. Gmitter and A. Scherer, "30\% external quantum efficiency from surface textured, thin-film lightemitting diodes", Appl. Phys. Lett., vol. 63, p. 2174$2176,1993$.

[4] H.W. Deckman and J.H. Dunsmuir, "Natural lithography", Appl. Phys. Lett., vol.41, p.377-384, 1982.

[5] C. Hilsum, Editor, Device Physics, Amsterdam: North-Holland, Handbook on Semiconductors, vol. 4, 1993, Chapter 7.

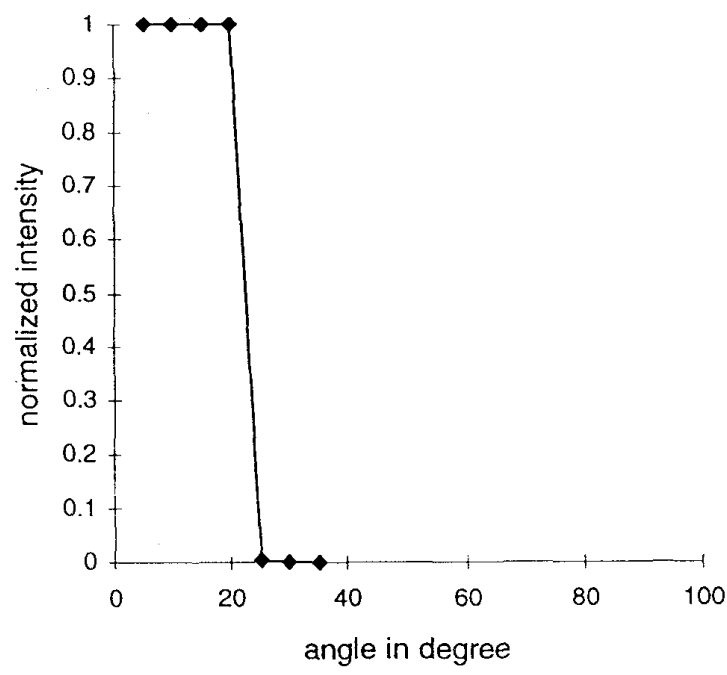

Figure 5. Radiation of a typical flat surfaced LED

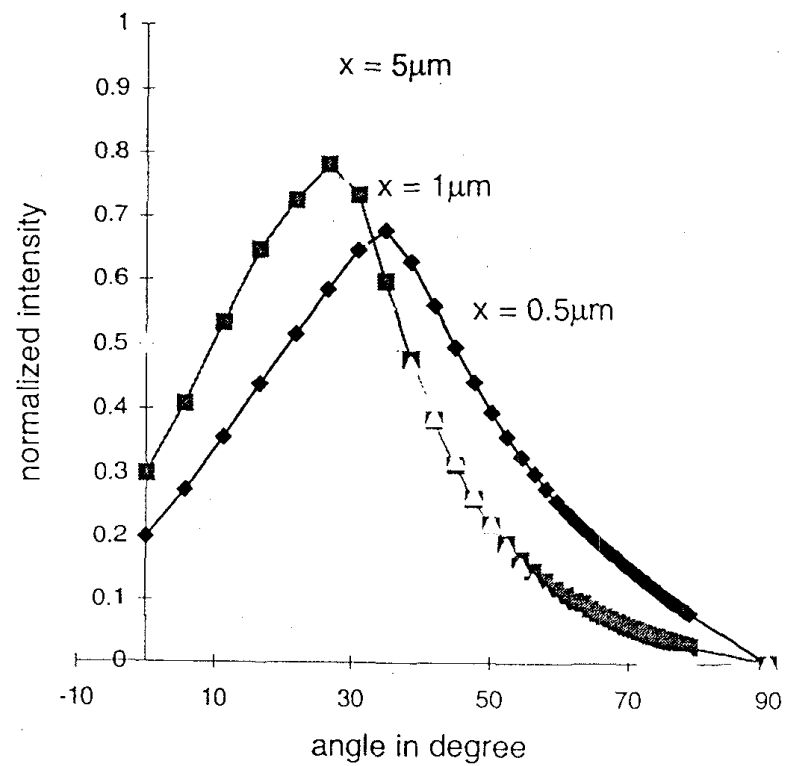

Figure 6. Radiation pattern of a textured surfaced LED 